\title{
Is there a propeller neutron star in $\gamma$ Cas?
}

\author{
M. A. Smith, ${ }^{1 \star}$ R. Lopes de Oliveira,${ }^{2}$ C. Motch $^{3}$ \\ ${ }^{1}$ National Optical Astronomy Observatory, 950 N. Cherry Ave., Tucson, AZ, USA \\ ${ }^{2}$ Universidade Federal de Sergipe, Departamento de Física, Av. Marechal Rondon, S/N, 49000-000 São Cristóvão, SE, Brazil; \\ Observatório Nacional, Rua Gal. José Cristino 77, 20921-400, Rio de Janeiro, RJ, Brazil \\ ${ }^{3}$ Université de Strasbourg, CNRS, Observatoire Astronomique, UMR 7550, F-67000, Strasbourg, France
}

Accepted XXX. Received YYY; in original form ZZZ

\begin{abstract}
$\gamma$ Cas is the prototype of a small population of B0-B1.5 III-V classical Be (cBe) stars that emit anomalous and hard X-rays with a unique array of properties. $\gamma$ Cas is known to host, like other cBe stars, a decretion disk and also a low mass companion. Recently Postnov et al. have posited that this companion is a magnetized rapidly spinning neutron star that deflects direct gravitational accretion from a stellar/disk wind via the "propeller mechanism." These authors state that the key X-ray observations are "remarkably well produced" in this scenario. We reexamine this mechanism in detail and conclude that there are a number of fatal objections in its application to the $\gamma$ Cas case. Among other considerations these issues include the prediction under the propeller scenario of a much smaller population of $\gamma$ Cas stars than is observed and the lack of allowance for observed correlations of X-ray and UV and/or optical properties over a variety of timescales.
\end{abstract}

Key words: Stars: individual - Stars: emission line, Be - X-rays: stars - Stars: massive - Stars: neutron - accretion, accretion disks

\section{INTRODUCTION}

Over the last twenty years we authors have conducted a number of observational campaigns to monitor the properties in the X-ray, optical, and ultraviolet domains of the hard X-ray emitter $\gamma$ Cas (B0.5 IV-Ve). All this work has led us to a picture in which these anomalous X-ray emissions are produced by the interaction of magnetic fields of this star and its Be "decretion" disk. This holds as well among "analog" members of this new X-ray Be subgroup.

In this picture $\mathrm{X}$-rays are generated from high energy particle beams directed to the surface of the Be star and depositing their energy as hard X-ray-visible quasi-flares. These beams are created by acceleration of particles situated within the ambient field lines following their entanglement of two magnetic field structures. The first structure consists of local chaotic field lines emanating from and corotating with the star. The second is a toroidal field embedded in the Be star's Keplerian disk and perhaps amplified by the Balbus \& Hawley (1991) magnetorotational instability (Robinson et al. 2002, "RSH02"). Robinson \& Smith (2000, "RS00") reported numerical simulations to show what attributes the high energy electron beam might have to generate the observed X-ray flux. Of course there is no way to directly observe this complicated interplay. Rather it can be

^ E-mail: myronmeister@gmail.com inferred only as a result of employing a variety of observational techniques.

In a recent paper Postnov et al. (2017, "POT17") have proposed instead that the hard X-ray emission from the $\gamma$ Cas system is caused by the operation of a "propeller" associated with a rigidly rotating magnetosphere around a putative neutron star (NS) secondary. an idea first raised in the context of $\gamma$ Cas long ago by Corbet (1996). However, this narrative has intrinsic weaknesses and overlooks key observational properties of the Be star and disk. We believe also the mechanism they propose is inherently untenable for these stars, and we address these issues herein. In doing so we occasionally draw on additional information known from other analogs of this $\gamma$ Cas subgroup. Much of this information is taken from a general review paper (Smith et al. 2016, "SLM16") and references cited therein.

\section{X-RAY AND RELATED PROPERTIES OF $\gamma$ CAS}

$\gamma$ Cas is the prototype of a subgroup of 10-12 Galactic X-ray classical Be stars. Members of this subgroup are confined to the region B0-B1.5, luminosity class III, IV in the HR Diagram (SLM16). RV studies of $\gamma$ Cas reveal it to be a single-line binary in a wide, circular orbit $(\mathrm{P}=203.59$ days; $e \leq 0.03$ (Smith et al. 2012a, "SLM12a"). The secondary's 


\section{M. A. Smith, R. Lopes de Oliveira, \& C. Motch}

mass is about $0.8 \pm 0.4 \mathrm{M}_{\odot}$. Otherwise, we stress that its evolutionary status is unknown. For example, the secondary could be a late-type main sequence star or for that matter a passive degenerate star. Long Baseline Optical Interferometry from a number of studies has fixed the obliquity of the Be-disk system to our line of sight as $i \approx 42^{\circ}$ (SLM12a). The Be component of $\gamma$ Cas has a rotational period of $\approx 1.22$ days (Henry \& Smith 2012, "HS12"). This conclusion comes from a robust periodic feature in its optical light curve and is consistent with the extreme rotational broadening of its spectral lines. Given this period, its estimated radius and sin i obliquity, $\gamma$ Cas appears to rotate nearly at the critical velocity. This rotation could produce localized magnetic dynamo instabilities due to an enhanced equatorial convection zone (Motch et al. 2015, "MLS15"). This may speak to the cause of the star's role in generating hard X-rays and can address the possible criticism that most other early Be stars with extensive disks do not emit this radiation. Likewise, it is sometimes speculated that $\gamma$ Cas is in an intermediate state of binary evolution because as many as three members of the subgroup could be blue stragglers in clusters (SLM16).

$\gamma$ Cas stars are recognizable by their unique array of Xray emission characteristics among high mass X-ray stars. The emission is "hard," and their moderate $L_{\mathrm{X}}$ luminosity, $3-10 \times 10^{32} \operatorname{ergs~s}^{-1}$, (as measured in the $0.1-10 \mathrm{keV} \mathrm{X}$-ray band) is intermediate between so-called classical Be stars and BeXR binary systems. High resolution spectra of $\gamma$ Cas, which include the continuum and resolve the Lyman $\alpha$ emission lines of Fe XXV (at $6.7 \mathrm{keV}$ ) and Fe XXVI (at $6.9 \mathrm{keV}$ ), are fit well with an optically thin thermal model described by a "primary" plasma temperature " $k T_{\text {hot }} \approx 14 \mathrm{keV}$ and (usually) an attenuation of soft X-ray flux by photoelectric absorption of "cold" matter. Importantly, for $\gamma$ Cas a second (often higher) column density of cold matter is also present in the foreground of the hot primary X-ray plasma. We should note clearly that the flux of the primary component dominates at all X-ray wavelengths (save the wavelengths of soft and moderate energy X-ray lines). It follows that the attenuation of soft X-ray signals the presence of this cold matter along the line of sight to the hot plasma behind it. It remains to be added that fluorescence lines of Fe and Si are present in the $\gamma$ Cas X-ray spectrum. These features, which change with time, indicate the presence of cold matter close to the hot X-ray sources.

The primary plasma can take on various $k T_{\text {hot }}$ values for different members of the $\gamma$ Cas subgroup. For at least half of those $\gamma$ Cas stars observed more than once the primary plasma's temperature has been found to change. In addition, for the best observed stars the presence of emission lines of $\mathrm{H}$ - and He-like lines of lower ionization metallic species at lower energies (0.3-0.6 keV band) discloses that 2-3 secondary cooler plasmas are present. These plasmas seem to be monothermal because a model described by a Differential Emission Measure, that is, by a plasma having by a continuous distribution of temperatures, does not give a good fit (Lopes de Oliveira et al. 2010), (SLM12a).

Although the abundances derived from spectral analysis are generally consistent with solar values, for a few elements their values are nonsolar and these abundances vary over time. These include over or underabundances of atomic neon and nitrogen. These anomalies are inconsistent with abundances produced by core or shell burning in late stages of stellar evolution, e.g., of a putative pre-supernova. Curiously, a persistent low iron abundance has been found from the Fe K-shell lines of Fe XXV and Fe XXVI determined by many authors using as many X-ray telescopes, even though the abundance derived from L-shell ion lines is solar-like.

The X-ray light curve of $\gamma$ Cas exhibits an array of variations characterized by certain timescales. These include (1) ubiquitous "shots" or quasi-flares lasting a (few seconds to a minute), (2) erratic "undulations" over 10 s of minutes to hours, (3) long-cycles ( $\sim 70$ days), and (4) seemingly chaotic, even longer term variations (SLM16, MLS15). In addition to the "flares," variations of an underlying "basal" X-ray flux component can be present. This component constitutes $\approx \frac{2}{3}$ of the total flux, exhibits the same $k T_{\text {hot }}$ as the flares, and varies on a timescale of a few hours (bullet 2). Unlike true magnetic flares in cool stars, the tails of the flare profiles exhibit no tapering or extensions (Smith et al. 1998a, "SRC98"). This fact suggests a rapid decay timescale. In general, the flares are visible in both soft and hard X-ray bandpasses, but exceptions occur in which they are present in one energy band and are weak or absent in the other according to Smith et al. (2012b, "SLM12b"). This study also found that the flare properties of $\gamma$ Cas are essentially the same for another $\gamma$ Cas analog, HD 110432 (B0.5 IIIe).

Power spectra of the short timescales (1) and (2) variations describe epoch-dependent, mild deviations from a $1 / f^{n}$ relation (where $n$ is always near unity). However, the details of these relations depend upon an epoch-dependent distribution of flare strengths.

The quasi-sinusoidal $~ 70$-day cycle was first observed in optical photometry. This cycle is almost ubiquitous and in the X-ray region attain an amplitude of a factor of 2-3. These X-ray variations generally correlate well with optical variations (RS00, HS12, MLS15). The Johnson $V$ band amplitudes of the optical cycles are greater than the $B$ ones. This means the only other possible contributor to red-optical flux in the Be complex, the Be decretion disk, must play an important role in the generation of at least the variable component of the hard X-ray flux. This is the primary reason for including the disk in the magnetic interaction picture.

On a timescale of several months, MLS15 found that optical flux changes caused by density variations in the inner part of the decretion disk are strongly correlated with X-ray fluxes with a similar flux ratio as seen for the $70 \mathrm{~d}$ cycles. They found that optical and X-ray fluxes vary together with a time delay of less than one month. These authors have also shown that such a small time lag is inconsistent with the expected transit time from inner disk regions to the orbit of the companion.

For completion, we note that UV light curves of $\gamma$ Cas exhibit small dips lasting a few hours, and optical helium line profiles exhibit blue-to-red rapidly moving migrating subfeatures (Smith et al. 1998b). Neither of these signatures is correlated with the star's X-ray flux, though both indicate the presence of plasma forced into corotation just over the star's surface.

Apart from the hard flux variations, the soft relative to hard energy flux can change on timescales of ten days or longer (SLM12a), and at times rapidly over several minutes (Hamaguchi et al. 2016). This is the "attenuation effect" noted above. The occurrence of this effect is important because it conveys important geometrical details of the hard 
X-ray sources. In this case SLM12a noted that the presence of intervening cold matter indicates no relation with binary phase of $\gamma$ Cas during an outburst event in 2010. Significantly, the cold matter absorption column increased simultaneously with the beginning of the 2010 Be outburst, which is to say the ejection of matter from the Be star. The occurrence of this column of the cold matter places the hot, high plasma density, X-ray sources between observer and the Be star. We develop this point below.

A particularly salient discovery concerning the hard Xray flux is the tight relation between its variations and ultraviolet line strengths and continuum, and optical light curves (SLM16 and references cited therein). For example, from a 21-hour simultaneous monitoring in 1996 of $\gamma$ Cas by the Rossi X-ray Telescope Explorer (RXTE) and the Goddard High Resolution Spectrograph (GHRS) attached to the Hubble Space Telescope, Smith \& Robinson (2003, "SR03") reported that increases in X-ray flux were associated with strengthenings of a UV Fe V line and weakenings of UV Si III lines on timescales of several minutes. This behavior is consistent only with plasma near a bright and optically thick medium, namely when the UV line strengths increase or decrease in response to a closeby X-ray source. These relationships constitute a particularly critical argument in locating the X-ray plasma near the Be star.

We underscore that observations reported in many studies of early-B/degenerate star binaries at large disclose that ultraviolet light from the secondary component is not visible against the primary's contribution. Even for systems with hot degenerate secondaries essentially all the UV flux comes from the B star. Also, while initial hydrodynamical simulations suggest that the disks are likely to be truncated by 3:1 orbital tidal resonances (Okazaki \& Negueruela 2001), the actual mass transfer rate deposited to the secondary (whether degenerate or otherwise) is unknown.

\section{PROPERTIES OF X PER}

In the POT17 scenario $\gamma$ Cas can evolve to become a $\mathrm{X}$ Per-like object. Therefore we summarize the relevant attributes of this well-known system. The Be binary Xray pulsar X Per/4U352+309 consists of an O9 III primary star and X-ray pulsing $\left(\mathrm{P}_{\text {puls }} \sim 837 \mathrm{~s}\right)$ NS. As summarized by Lutovinov et al. (2012, "LTC12"), the system has a low/intermediate orbital eccentricity $e=0.11$ and $\mathrm{P}_{\text {orb }}=$ 250 days. The X-ray luminosity $(0.1-10 \mathrm{keV})$ at its usual low state is about $2 \times 10^{34} \mathrm{ergs} \mathrm{s}^{-1}$. LTC12 found that during one of its X-ray outbursts its X-ray flux increased and then subsided by a factor of five. The rise and decline each lasted a year or so while its optical light curve showed little or no response. As already noted, in contrast the largest Xray variations of $\gamma$ Cas occur in the form of its rapid "flares." Its longer term variations do not exceed a factor of 2-3 at most, and these are correlated with optical flux. X Per is the prototype of a small group of "persistent" Be slow X-ray pulsar systems. These persistent systems are generally thought to be products of "low kick" orbital perturbations resulting from a mild SN explosion.

The X-ray emissions from a Be-NS system are determined by details of a wind flow from the Be star (or its disk) to a magnetized NS companion. ${ }^{1}$ Magnetic field strengths of these pulsars are estimated to be of the order $10^{12} \mathrm{G}$ or even higher. Particles from the wind are ultimately deposited onto the surface of a magnetosphere (defined below), where they are guided into columns by the pulsar's field toward its magnetic poles. We observe the X-ray emission along these columns as pulses because the poles are rotationally advected across our line of sight. The resulting spectrum is typically optically thick. In the case of X Per the spectrum can be fit well at high energies with a hard power law (index $n \approx 2$ ) and at low energies by a relatively cool blackbody (e.g., LTC12). These attributes are very different from those in the $\gamma$ Cas spectrum.

\section{THE PROPELLER MECHANISM}

In describing the geometry of particle accretion onto X-ray pulsars we should first summarize the properties of three characteristic radii of volumes around the NS. The first is the well known Bondi-Hoyle radius, $R_{B}$, within which wind particles from the Be star or its disk spiral toward the NS due to gravitation. They will deposit their energy on the star's surface as X-rays or in its magnetosphere if the NS is highly magnetic.

The second important size is of the magnetosphere, given by the Alfven radius $R_{A}$. This is the distance from the NS for which the decreasing magnetic pressure is balanced by the ambient ram pressure from the wind. Inside this radius the magnetic pressure wins and so the magnetosphere rotates rigidly with the NS and carries along embedded plasma. In the simple dipole case captured wind particles flow mainly toward the magnetic poles.

The remaining important radius is the corotation radius, $R_{C}$, which is the point at which the angular velocity of particles orbiting the star equals the NS rotational value. Inflowing magnetically channeled wind particles just beyond this radius are super-Keplerian and spiral outward to a larger orbit.

For typical Be-magnetic NS systems the Alfven radius lies interior to the corotation radius. This permits wind particles to freely transit from their origin to the rotating magnetosphere and be captured by magnetic stresses at its surface. The kinetic energy of the particles is transformed chiefly to X-rays, which typically display a power-law (nonthermal) spectrum. The particles are channeled along magnetic flux lines toward the NS. The luminosity $\mathrm{L}_{x}$ depends in part on the accretion radius, which in this case is the much larger Bondi radius, $R_{B}$.

For the case of $\gamma$ Cas POT17 have considered an alternative propeller regime, for which $R_{C}<R_{A}$, which may occur for fast rotating pulsars. In this configuration the rigidly rotating magnetosphere extends to a region where wind particle velocities are super-Keplerian, they can fall no further than the magnetosphere's boundary. The centrifugal forces at the surface of the magnetosphere prevent particle accretion such that most of them are deflected outwards. They subsequently accumulate within a shell bounded by $R_{A}<r$

1 We use the term "wind" throughout, in the understanding that the outflow may not be due to the usual line-driven radiative wind occurring in most OB stars (see e.g., Carciofi et al. 2012). 
$<R_{B}$, with most of them concentrated at the inner boundary. The particles are then pushed by the upstream ram pressure of the wind deep into the magnetosphere. The surface shell remains optically thin and so it emits little UV or optical flux. The accretion radius (and hence $\mathrm{L}_{x}$ ) and temperature of the heated plasma is now partly determined by $R_{A}$, which in turn is much smaller than $R_{B}$. Notice that a substantial X-ray luminosity and hard spectrum can result. A number of papers in the literature have suggested that the outburst or high state of short period BeXR systems occurs when the propeller mechanism temporarily ceases to operate and uninhibited accretion resumes (see e.g. Tsygankov et al. 2016; Christodoulou et al. 2016; Reig \& Milonaki 2016, and references therein). These outburst events occur on short timescales, at least in part as response to surges in wind density, leading to brief outbursts from direct accretion. Since $R_{A}$ shrinks in response to increases in accretion density, the inequality is reversed, that is $R_{A}<R_{C}$, during these outbursts. Again, this outburst behavior is in contrast to long term changes in the accretion phase forced by NS spin-down and binary evolution. These evolutionary considerations are discussed in the next section.

\section{EVOLUTIONARY TIMESCALES}

The POT17 proposal merges the above ideas into a putatively integrated evolutionary scenario. These authors pictured $\gamma$ Cas as a progenitor of a class of low to moderate eccentricity BeXR systems such as X Per. In their description of the $\gamma$ Cas system the putative fast rotating NS is in the propeller mode and gradually loses rotational energy owing to a braking torque of the surrounding magnetosphere. This torque acts from radiative losses and mechanical drag on the rotating magnetosphere by the wind. As the rotational angular velocity of the NS slows, the corotation radius $R_{C}$ increases, moves outside $R_{A}$, and direct accretion via gravitation can start. POT17 estimated that the propeller phase could last "several $10^{5}$ years or even longer." Next POT17 advanced several predictions to adduce the applicability of their picture.

We will comment on the POT17 predictions and as well as on the applicability of their scheme in $\S 6$. We review first the durations of the so-called ejector and propeller phases of the magnetic neutron star. These durations determine the sizes of the population of $\gamma$ Cas-type systems, which one can compare with the number of known systems in our Galactic neighborhoods. In $\$ 5.2$ we will present a more complete evolutionary scheme than did POT17 for the transfer of wind particles to the putative NS.

Our scheme has the sequence:

ejector $\rightarrow$ propeller $\rightarrow$ direct accretion $\rightarrow$ spinequilibrium.

The ejector phase will be discussed in \$5.2.1. As implied, "direct accretion" here simply refers to the flow of wind particles to the surface of the NS in the case of no (or weak) magnetic field; as noted by POT17 the resulting $\mathrm{L}_{x}$ can be very high because the full gravitational energy of the former wind particles is liberated near the small NS radius. Spin equilibrium, which perhaps should be more accurately called a "quasi-equilibrium," occurs when the Keplerian orbital velocity of the outer edge of the magnetospheric disk equals the corotation velocity at that point, as defined by Waters \& van Kerkwijk (1989). The X-ray source then resides on the Corbet relation for BeXR systems (Corbet 1984). Also note that if the accretion rate changes rapidly the NS system can transition back and forth between the propeller and ejector modes on short timescales.

\subsection{The velocity of the accreted material}

The mass accretion rate required to feed the NS in the propeller mode contrains the possible range of velocities of the accreted material at the Bondi radius. Importantly, as noted by POT17 the duration of the propeller phase critically depends on this velocity.

According to Eq. 15 of POT17, the mass accretion rate required to sustain an X-ray luminosity of $L_{32} \times 10^{32} \mathrm{erg} \mathrm{s}^{-1}$ on a neutron star in propeller mode is

$\dot{M}_{\mathrm{x}} \approx 8.3 \times 10^{-11} \mu_{30}^{2 / 15} L_{32}^{4 / 5} M_{\odot} y r^{-1}$,

where $\mu_{30}$ (which is $\mu /\left(10^{30} \mathrm{Gcm}^{3}\right)$, the typical dipole magnetic moment of the neutron star, is approximately unity, and assuming that the mass of the NS, $M_{\mathrm{X}}$ is $1.4 M_{\odot}$. The latter is larger than the $1.0 M_{\odot}$ value taken by POT17 and is a more realistic value, as noted below; see also Ozel et al. (2012); Ozel \& Freire (2016). Since accretion occurs though wind capture $\dot{M}_{\mathrm{x}}$ is the Bondi accretion rate and $\dot{M}_{\mathrm{X}}=\pi \rho(r) V_{\mathrm{O}} R_{\mathrm{B}}^{2}$, with the Bondi radius expressed as $R_{\mathrm{B}}=2 G M_{\mathrm{X}} / V_{\mathrm{O}}^{2}$. Here we have retained the POT17 notation for parameters. This includes $V_{\mathrm{O}}$ for the vectorial sum of the wind and orbital velocities to which should be quadratically added the sound speed in the accreted material $\approx 10 \times\left(T / 10^{4} K\right)^{1 / 2} \mathrm{~km} \mathrm{~s}^{-1}$. Our notation for parameters of the Be star will be an asterisk.

Assuming that the neutron star accretes matter from the intermediate latitude wind that is typical of an early B star, we can relate the mass accretion rate onto the NS to the total mass loss of the Be star. With $M_{*}=15 M_{\odot} ; M_{\mathrm{x}}=$ $1.4 M_{\odot}$ and $P_{\text {orb }}=203.59 \mathrm{~d}, M_{*}$, the total mass loss rate of the primary wind, should be

$\dot{M}_{*}=1.2 \times 10^{-4}\left(\frac{V_{\mathrm{o}}}{1000 \mathrm{~km} \mathrm{~s}^{-1}}\right)^{4} M_{\odot} y r^{-1}$,

which is a few orders of magnitude above the total wind mass loss rate expected for a B0 IV star, $\dot{M}_{*} \sim 10^{-8} M_{\odot} y r^{-1}$.

From the foregoing, one can see that in order to capture enough material the orbit of the neutron star has to be nearly coplanar with the plane of the decretion disk and accrete low relative velocity and high density matter. The fraction of observed Be-shell stars led Porter (1996) to conclude that the disk opening angle is typically $\approx 5$ degrees, although disk flaring at large radii may weaken this constraint.

The range of observationally permitted orbital inclinations depends on the assumed masses of the B0 star and NS. Assuming the values given above for the primary and secondary stars, combined with the observed radial velocity semiamplitude of $3.8 \mathrm{~km} \mathrm{~s}^{-1}$ (SLM12a), implies a low orbital inclination on the order of $i \approx 29^{\circ}$, which is at variance with that of the decretion disk $\left(i=42^{\circ}\right)$ derived from optical interferometry (SLM12a). Such a significant misalignment of the NS orbit with the disk plane can be expected to drive an X-ray flux modulation at the orbital period. 
POT17 argue that the helium star electron capture channel could yield neutron stars with masses as low as $1 M_{\odot}$. In this case, a primary with a somewhat high mass of $16 M_{\odot}$ would indeed allow a $1 M_{\odot}$ mass NS (their assumed mass values) to orbit close to the plane of the decretion disk. However, there is no observational evidence of such low mass $\left(\leq 1 M_{\odot}\right)$ neutron stars. Although rather few reliable NS mass estimates exist for Be/X-ray systems, not to mention $\gamma$ Cas-like systems, masses of the non-recycled $\mathrm{NS}+\mathrm{WD}$ or NS+NS binaries - descendent of these systems do not show evidence of a significant population of low mass neutron stars (see e.g., Ozel \& Freire 2016). In addition, the amount of accreted matter during the putative X-ray active stage of a few $10^{6} \mathrm{yr}$ is only $10^{-3} M_{\odot}$ at most. Therefore we will take the mass of the NS as constant over the entire period.

Optical spectroscopic observations of Be stars have established that the decretion disk is essentially Keplerian, with no detectable outflow velocity (see e.g. Stee et al. 2012, and references therein). As already noted, in low eccentricity $\mathrm{Be} / \mathrm{X}$-ray binary systems such as $\gamma$ Cas the disk is very efficiently truncated at the $3: 1$ resonance radius, leaving a wide gap size between the edge of the disk and the neutron star (Okazaki \& Negueruela 2001). Additional numerical simulations by Okazaki \& Negueruela (2001) suggest that the infalling disk matter acquires velocities relative to the neutron star comparable to the sound speed and are at most of the order of a few tens of $\mathrm{km} \mathrm{s}^{-1}$. For these velocities the Bondi accretion radius is comparable to the radius of the Roche lobe. At the distance of the Roche radius of the accreting object in $\gamma$ Cas, the free-fall velocity is $\approx 80 \mathrm{~km} \mathrm{~s}^{-1}$. Whatever the range of primary and secondary masses considered, the orbital velocity of the accreting object remains at nearly similar values of the order of $80-90 \mathrm{~km} \mathrm{~s}^{-1}$ for low eccentricities. It is therefore unlikely that the $\mathrm{V}_{0}$ velocity in Eq. 14 of POT17 reaches values much above $100 \mathrm{~km} \mathrm{~s}^{-1}$. Accordingly, durations of the propeller phase in excess of a few $10^{5}$ yrs are very unlikely.

\subsection{Spin period evolution}

\subsubsection{Ejector phase}

The very first phase of the neutron star history is the socalled ejector phase during which the ram pressure of the wind entering the gravitational influence of the neutron star at the Bondi radius is lower than the outgoing flux of electromagnetic waves and relativistic particles emitted by the magnetic neutron star (pulsar) (see e.g., Popov \& Turolla 2012, "PT12"). This condition is expressed as $P_{\mathrm{d} y n} \leq P_{\mathrm{PSR}}$, with $P_{\mathrm{d} y n}=\rho(r) V_{\mathrm{o}}^{2}$ and $P_{\mathrm{PSR}}=\dot{E} /(4 \pi R c)$. The power radiated by the slowing down pulsar due to dipole radiation is

$\dot{E}=8 \pi^{4} B^{2} R_{\mathrm{n} s}^{6} \sin ^{2} \alpha /\left(3 c^{2} P^{4}\right)$,

where $B$ is the magnetic polar dipole field, $R_{\mathrm{X}}$, the radius of the neutron star and $\alpha$ the inclination of the magnetic dipole from the rotation axis.

When does this ejector phase end? This question can be addressed by first assuming the POT17-adopted values of density and velocity for the incoming matter at the Bondi radius sufficient to explain the observed X-ray luminosity in the propeller mode (eq. 1), and also by using PT12's Eq. (1). We can then determine the spin period $P_{\text {ee }}$ at which the ejector mode ends. This is

$P_{\mathrm{ee}} \approx 0.48 \times\left(\frac{V_{\mathrm{o}}}{100 \mathrm{~km} / \mathrm{s}}\right)^{-1 / 4}\left(\frac{B}{10^{12}}\right)^{1 / 2} L_{32}^{-1 / 5} s$,

assuming $M_{\mathrm{X}}=1.4 M_{\odot}$ and $\alpha=90$ degrees.

Next, following PT12, and assuming a canonical moment of inertia $I=2 / 5 M R^{2}$ equal to $10^{45} \mathrm{gm} \mathrm{cm}^{2}$, the duration of the ejector phase may be computed as

$\tau_{\mathrm{ej}} \approx 3.6 \times\left(\frac{V_{\mathrm{o}}}{100 \mathrm{~km} / \mathrm{s}}\right)^{-1 / 2}\left(\frac{B}{10^{12}}\right)^{-1} L_{32}^{-2 / 5} M y r$.

Assuming factors of order unity for parameters in eq. $5, \tau_{\mathrm{ej}}$ should be a few million years. In addition, the putative neutron star should have spun down already significantly during the ejector phase.

\subsubsection{Propeller phase}

As implied in $\S 4$, the end of the propeller accretion mode occurs when the corotation radius, ever increasing because of NS spin-down, reaches value close to the magnetosphere radius. Using Eq. 7 of POT17 for the magnetosphere radius and assuming a $1.4 M_{\odot}$ neutron star implies that the high $\mathrm{X}$-ray luminosity accretion mode switches on as soon as the spin period becomes longer than

$P_{\mathrm{e} p}=8.6 \times \mu_{30}^{4 / 5} L_{32}^{-1 / 5} s$.

The braking torque acting on the neutron star in the propeller mode is given by Eq. 12 in POT17. Importantly, the torque is constant and does not depend on the difference between the critical and actual angular frequencies. Accordingly, the duration of the propeller phase is of the order of $\tau_{\text {prop }}=2 \pi\left(1 / P_{\text {ep }}-1 / P_{\text {ee }}\right) / \dot{\omega}$, with

$\dot{\omega}=-49 I^{-1} \omega_{\mathrm{B}}^{2} r_{\mathrm{B}}^{3} C \frac{R_{\mathrm{A}} L_{\mathrm{X}}}{G M_{\mathrm{X}} V_{\mathrm{o}}}$,

with $\omega_{\mathrm{B}}$ the orbital angular frequency and $R_{\mathrm{A}}$ the magnetosphere radius. The duration of the propeller phase is given by POT17 in their Eq. 14. It should be reiterated that some significant spin-down will have occurred during the relatively long ejector phase. Here we use $P_{*}=0.48 \mathrm{~s}$ as derived in our eq. 4 , a value much longer that the probable birth spin period. Importantly, this significantly further shortens the duration of the propeller phase. Using the Alfven radius in their Eq. 7, an average X-ray luminosity of $5 \times 10^{32} \mathrm{erg} / \mathrm{s}$ (SLM16) and $P_{\mathrm{orb}}=203.5 \mathrm{~d}$, we can express the actual duration of the propeller phase as

$\tau_{\text {prop }} \approx 5 \times 10^{5}\left(\frac{V_{\mathrm{o}}}{100 \mathrm{~km} / \mathrm{s}}\right)^{7} y r$

Note that this timescale is consistent with our estimate in $\S 5.1$, based on the range of velocities discussed there, and that it is several times shorter than the duration of the ejector phase given in eq. 5 . 


\section{OUR VERDICT: NO PROPELLER FOR $\gamma$ CAS}

\subsection{Propeller systems in context of Be/X-ray binary evolution}

Several independent evolutionary arguments just discussed suggest that the propeller mechanism as proposed by POT17 cannot account for the space density of the $\gamma$ Cas X-ray phenomenon.

First, as noted in $\$ 5.1$ the velocity of the accreted material at the Bondi radius is unlikely to be much greater than $\approx 100 \mathrm{kms}^{-1}$. As we have seen, the velocity of the dense flow extracted from the outer edge of the decretion disk probably does not reach values larger than a few tens of $\mathrm{km} \mathrm{s}^{-1}$. In addition, we have shown that a large part of the neutron star spin-down may have already occurred during the ejector phase. From these considerations one can see that both parameters $P_{*}$ and $V_{O}$ in Eq. 14 of POT17 probably have values consistent with a propeller phase duration of only on the order of a few $10^{5} \mathrm{yr}$. It seems clear that the steep dependency of $T_{\text {prop }}$ on relative velocity disallows the conclusion that the duration of the propeller phase can be as high as 1 Myr. Second, although there may be a few systems in the propeller phase, we consider it unlikely that a large population of NS $/ \gamma$ Cas-like propeller systems exists in the Galaxy. This is because of the rather short duration of the propeller phase. We develop this point in the following.

Over 100 HMXBs are known in the Galaxy, among which $\approx 80 \%$ are confirmed or candidate Be/X-ray systems (Liu et al. 2006; Reig 2011). HMXB population models, e.g., by Portegies Zwart \& Verbunt (1996, "PV96"), Shao \& Li (2014, "SL14") predict the existence of $\approx 500$ Be/X-ray binaries in the Galaxy - a figure roughly consistent with the number of systems known, taking into account observational biases. With a formation rate of $\approx 5 \times 10^{5} \mathrm{yr}^{-1}$, including effects of NS birth kicks, the lifetime of X-ray Be systems is of the order of $\approx 10 \mathrm{Myr}$ (PV96).

Given the observed long Be/X-ray phase, comparable to the evolutionary time scale of an early type mass donor star, the number of progenitors of putative $\gamma$ Cas propellers cannot be much higher than that of directly accreting systems. Consider therefore the following contradiction. Apart from a handful of nearby systems detected in low sensitivity all-sky surveys (HEAO-A1 and Rosat all-sky), the great majority of $\gamma$ Cas-like systems were discovered in XMM-Newton galactic surveys (SLM12a). Only a few percent of the Galaxy have been observed by XMM-Newton, and fewer still have been followed up in optical spectroscopy. Nebot et al. (2013) report the discovery of 4 new $\gamma$ Cas-like objects at distances of $\approx 2 \mathrm{kpc}$ in a $4 \mathrm{deg}^{2}$ survey. Taken at face value, and even considering larger errors, this implies the presence of several thousand $\gamma$ Cas-like objects in the Galaxy, a figure much larger than the 500 members predicted by PV96 and SL14 for the entire set of Galactic Be/X-ray binaries.

Conversely, one may compare the frequency of systems in the ejector phase to systems in the propeller phase. Collision of the relativistic pulsar wind with the stellar wind generates copious high energy emission across the X-ray to the $\gamma$-ray regime (see e.g., Bogovalov et al. 2008). The spectral energy distribution of the binary Be/radio pulsar binary PSR B1259-63 (so far the only one known) peaks in the 10$100 \mathrm{MeV}$ energy range and extends up to more than $100 \mathrm{GeV}$
(Abdo etal. 2011). Dubus (2013, "D13") convincingly argue that most $\gamma$-ray binaries are made of young radio pulsars embedded in the circumstellar material of a massive star. Only pulsars with spin-down luminosities above $10^{35} \mathrm{erg} \mathrm{s}^{-1}$ are energetic enough to produce $\gamma$-rays, implying $\gamma$-ray life times of the order of $6 \times 10^{5} \mathrm{yr}$ (D13). ${ }^{2}$ Even given the attendant observational biases, the low observed frequency of Galactic $\gamma$-ray binaries (only this one Be/NS binary is known in the $\gamma$-ray active ejector state) is consistent with the population of their wind-accreting BeXR binary descendants. This provides further support for the conclusion that the propeller mechanism is unable to explain the number of $\gamma$ Cas stars observed.

POT17 further assume that all $\gamma$ Cas-like systems must have a low eccentricity, due to the low kick velocity imparted by the particular supernova mechanism assumade. However, there is no evidence for such a large population of systems existing before the propeller phase (ejectors), nor afterwards (classical Be/X-ray binaries). In particular, all $\gamma$-ray ejector binaries have very eccentric orbits $(e \geq 0.35$, including PSR B1259-69). In addition, all young radio pulsars in binaries with massive companions that have terminated their $\gamma$-ray active phase but are still in the ejector phase also have very high eccentricities ( $e \geq 0.58$; Manchester (2005, "M05"), D13) and spin-down times $\left(\tau_{s d} \approx 3-5 M y r\right.$; M05), very consistent with the duration of the ejector phase computed in eq. 5. The range of eccentricities observed in post-SN systems is consistent with that observed in Be/X-ray binaries in which the vast majority of the systems have eccentricities $\geq 0.3$ (Reig 2011). In addition, the great majority of Be-slow pulsar systems like X Per have noncircular orbits. Indeed, in the sample of eight long-period pulsar systems identified by Knigge, C. et al. (2012) none is in an almost circular $(e \leq 0.03)$ orbit. If such systems are in noncircular orbits, while $\gamma$ Cas is not, then these two types of systems would appear to be members of two distinct populations.

Apart from the $\gamma$ Cas stars, there is no reason why a long-lived propeller stage could not exist in Be-NS binaries with larger eccentricities or inclined orbits as progenitors of the bulk of Be/X-ray systems.

\subsection{Our primary nonevolutionary objections to the Postnov et al. scenario}

POT17 do not address the critical details of the optical-UV$\mathrm{X}$-ray variations or of the X-ray flares. In fact, references in their paper to "ultraviolet" or "spectroscopic variations" were not made and apparently not considered. As outlined above, the correlation and anti-correlation of various UV spectral lines and UV continuum with X-ray variations means that the X-ray emitting plasma is strongly influenced by the only nearby major source of UV and optical wavelength flux, the Be star. POT17's overlooking of this fact is our first major objection, apart from evolutionary issues just discussed.

Second, the POT17 description of what we call flares (or "shots") was characterized as merely the short timescale end of a continuous distribution of variabilities. In fact, the

2 However, even for these systems the NS star remains $\gamma$-ray quiet during the ejector phase. 
short flare decay rates are critical to a more focused consideraton as they necessarily imply particle densities of up to $10^{15} \mathrm{~cm}^{-3}$ for the pre-flare parcels (SRC98). Such high densities are hardly characteristic of POT17's hot shell but they are characteristic of the Be star's lower atmosphere, and realistically only of this site. Insofar as these observations are not addressed, the POT17 picture is lacking.

Third, the correlation of optical and X-ray $~ 70$ day cycles mentioned above for $\gamma$ Cas has important implications. The existence of the red-tinged optical variation implies oscillations with a source that is cooler than the surface of the Be star yet still competing in its optical radiation with the Be star. The inner part of the decretion disk alone qualifies as the secondary source of red-optical light. This means the dense inner region of the disk must somehow be associated with the creation of most or all of the hard X-ray flux.

Fourth, in 2010 the soft-X-ray flux of $\gamma$ Cas decreased relative to its hard flux. This occurred during a 44 day monitoring period that coincided with an optical outburst. This event added matter to the line of sight column density. In other words, the hard X-ray source (which produces most of the soft X-ray flux as well) must lie close to the outbursting Be star. We stress here that the hot sources cannot be placed anywhere else but on the surface of the Be star - for example, in the inner disk as the density is too low to be consistent with the short flare decays mentioned above.

Fifth, POT17 claimed the prediction of a 40-day time lag of X-ray behind optical signal and cited a MLS15 result as confirmation. This is a misreading. Firstly, MLS15 found no lag at all. The "one month" figure MLS15 quote refers to a generous upper limit, not an equality. Secondly, the optical/X-ray time for any lag in Be-NS X-ray systems is not only the free-fall time into the NS potential well but must also include the transit times from the stellar ejection and transits through the Be disk and to the secondary companion. One must look to actual empirical examples of a transit time. A literature search for such correlated outbursts by MLS15 reveals two cases of optical-X-ray lag (one of them is X Per itself). The optical/X-ray lags in these cases, that is from the Be outburst to a response at the NS, have been observed to be about 4 years in both cases, (e.g., Haubois et al. 2012; Carciofi et al. 2012). Thus, this is the lag timescale one can expect under the POT17 or other accretion scenarios. It is in disagreement with the observational result of MLS15, which again pointsto the X-ray emission of $\gamma$ Cas arising from the vicinity of the Be star.

Any one of these arguments is sufficient to vitiate, or at the very least seriously question, whether the propeller mechanism is applicable to the $\gamma$ Cas stars.

\subsection{Other criticisms of the Postnov et al. scenario}

To complement the foregoing, we rebut purported predictions made by POT17:

- The propeller/hot shell picture predicts a continuous range of temperatures for the heated plasma from an initial high value, $k T_{\text {hot }}$ (POT17, eq. 3 ). Although we do not understand ourselves the origin of the secondary plasma components surrounding $\gamma$ Cas the evidence for their existence as discrete structures (e.g., SLM12a, SLM12b) contradicts the finding by POT17 that the temperature distribution should be continuous.

- The value of $\mathrm{kT}_{\text {hot }}$ derived by POT17 for the magnetosphere base is quoted as $\approx 27-32 \mathrm{keV}$. Since this figure is actually based on a selection of particular NS mass and radius values by the authors among a range of possible values, it is not necessarily a prediction. The stated temperature also depends upon the unknown mass flow rate impacting the Bondi sphere. Moreover, although a field strength of $10^{12} \mathrm{G}$ is generally quoted, the dispersion of these strengths among $\mathrm{X}$ Per-like pulsars is not well known. Given these uncertainties we doubt that the temperature according to their propeller model can be reliably predicted.

- Similarly, we note that two propeller systems, $4 \mathrm{U} 01165+63$ and V0332+53, that have been caught transitioning from a nonpropeller to propeller phase, exhibit soft, not hard, X-ray spectra during the latter phase (Tsygankov et al. 2016). This is obviously contrary to expectation from the POT17 model.

- POT17 discuss a "continuous" distribution of variability timescales from several seconds to a few days in $\gamma$ Cas's $\mathrm{X}$-ray light curve. However, this is somewhat misleading. Although periodograms of X-ray variations for $\gamma$ Cas indeed vary monotonically over these timescales, a detailed inspection of several data series reveals departures from a simple or smooth frequency pattern, and the degree of these departures varies from epoch to epoch (e.g., RS00). Importantly, POT17's discussion implies that the rapid variations are part of a chaotic pattern of variations. This characterization is in stark contrast to their character as isolated, plainly visible sharp features in all high resolution time series. As noted already, for timescales $>1$ day there is also a significant peak due to the $\sim 70$ day cycles in the low frequency region of the periodogram, Additional signal extends up to about one year.

- POT17 estimate an Emission Measure (EM) of $\approx 3.7 \times 10^{54} \mathrm{~cm}^{-3}$ for $\gamma$ Cas from their model. Without indicating whether more optimistic selections of parameters are still reasonable, they added that the EM can be some ten times higher. Available spectroscopic analyses of $\gamma$ Cas indicate EM values of up to $3 \times 10^{55} \mathrm{~cm}^{-3}$ (S04, SLM12a), or ten times above the best POT17 model. Similar values are obtained for HD 110432 (Torrejón et al. 2012; Lopes de Oliveira et al. 2010, "LSM10").

- POT17 estimated the convective velocity of $1000 \mathrm{~km} \mathrm{~s}^{-1}$ in their hot shell and they averred this to be an important prediction of X-ray line broadening. Actually, typical turbulence values measured in lines in high resolution X-ray spectra of $\gamma$ Cas are $300-500 \mathrm{~km} \mathrm{~s}^{-1}$ (S04 and SLM12a). POT17 overlooked these findings. The signal to noise ratio in $\mathrm{Fe}$ lines is generally too poor to make a reliable determination (LSM10), and interpretational issues occur for these lines as well. Also, SRC98 showed that velocities of $\gtrsim 1300 \mathrm{~km} \mathrm{~s}^{-1}$ (the expulsion as well as thermal velocity) could be predicted in their description of exploding surface flare parcels.

- POT17 state that the observation of the Fe fluorescent feature is another prediction of their picture. However, such features are present in many types of X-ray binary systems, regardless of the status of a degenerate companion and X-ray generation process.

- An important characteristic of the POT17 model is that the orbital and Be disk planes are aligned. As noted in $§ 5.1$, a 
significant tilt of the orbital plane would cause an X-ray flux modulation. Such a modulation is not seen (MLS15). Even a small kick may well move the NS out from its former orbital plane. Notice that there is no reason why a kick experienced by a NS explosion should be directed within the orbital plane. Since the POT17 hypothesis requires that all $\gamma$ Cas systems have propellers, it follows that the kicks of all of them be confined to their NS's equatorial planes. This would be a highly unlikely series of events.

- A salient feature of $\gamma$ Cas-like stars is their narrow range of X-ray luminosities. This would imply in the Postnov et al. scenario that in all $\gamma$ Cas systems, the NS should orbit at nearly the same distance from the Be star. Their decretion disks should also have similar extents and densities. We have already discussed already the improbable small ranges in orbital eccentricity and inclination implied.

- In the HR Diagram the $\gamma$ Cas stars are confined approximately to spectral types B0-B1.5 and luminosity classes III-V. One can expect their progenitors on the ZAMS to be late-type $\mathrm{O}$ stars. However, the domain of the persistent BeXR NS systems is somewhat larger. The persistent systems could not be expected to evolve to as narrow a spectral type domain as the $\gamma$ Cas stars occupy.

\section{FINAL CONSIDERATIONS}

In contrast to the propeller mechanism proposed by POT17, RS00, SLM16, and other studies cited therein have led to the interpretation that the generation of hard X-rays from $\gamma$ Cas are caused by an interaction of magnetic fields from the Be star and its decretion disk. Our initial picture has evolved with the accumulation of new datasets and is particularly informed by analyses of data from a number of multiwavelength observational campaigns. As noted, the light curves and spectra we analyzed consist of simultaneous X-ray/UV from the 1996 campaign, as well as some 19 seasons of robotic two-color APT photometry, most of which were contemporaneous with $\mathrm{X}$-ray monitorings. The latter include the long term RXTE All Sky Monitor program. An assessment of these combined datasets demonstrates that it is futile to construct any paradigm for the origin of the hard X-ray that does not include detailed analysis of concomitant optical and UV variations.

As noted by POT17, no magnetic signatures have been reported by spectropolarimetry in $\gamma$ Cas. It is very difficult to detect spectropolarization signatures in the very broadened lines of this star. Moreover, polarimetric techniques are tailored to the detection of magnetic dipoles, and other aspects of the star's behavior strongly suggest that any surface field cannot have a simple and hence easily detectable topology. Indeed, if the topology were simple the star's UV lines would be expected to show variations characteristic of a magnetic Bp star, and they do not. In recent years the periodic magnetic signature in the star's optical light curve has disappeared (HS12), rendering a polarimetric nondetection moot at the present time.

POT17 characterize the magnetic interaction scenario for $\gamma$ Cas as "entirely phenomenological at present and lacking in predictive power." Actually, this is not quite true. For instance, in our interaction picture hard X-ray production would cause clear changes in the attributes of the hard Xray production, such as a disappearance of flaring, the value of $\mathrm{k} T_{\text {hot }}$, abundances determined from X-ray lines, and the Fe K fluorescence feature. The optical/UV migrating subfeatures and UV "dips" might disappear as well. The larger point is that the interaction picture connects the inner disk conditions explicity with the X-ray production. Since the disk disappeared already in the early 20th century, we can look forward to it to disappear again and such tests to proceed. Otherwise, it is certainly true that continued observations have continually brought new surprises and necessitated changes to any posited model for the hard X-ray generation.

In all, we would say the "phenomenological" aspect of our magnetic interaction scenario is an expression of its adherence to a broad array of multiwavelength observations as well as being internally consistent. In fact, it seems as fair to characterize the POT17 evolutionary scenario as being dependent on critical assumptions about the mass loss rate of the Be/Be-disk system (by hypothesis the "disk wind") and the amount of mass available for accretion onto a degenerate companion. POT17 and we agree that this rate is not well known - indeed scant progress has been made on this hard to determine parameter.

We believe the POT17 propeller scheme fails on the basis of the many considerations we have discussed.

\section{ACKNOWLEDGEMENTS}

The authors wish to thank Dr. Chris Shrader for several edifying discussions. We also heartily thank comments by the referee, Dr. Dimitris Chrisodoulou, for clarifying important details pertaining to the propeller mechanism. R.L.O. was supported by the Brazilian agencies CNPq (Universal Grants 459553/2014-3 and PQ 302037/2015-2) and INCTA (CNPq/FAPESP).

\section{REFERENCES}

Abdo, A. A., Ackermann,M, Ajello, M., et al. 2011, ApJ, 736l, $11 \mathrm{~A}$

Balbus, S. \& Hawley, J. F. 1991, ApJ, 376, 214B

Bogovalov, S. V., Khangulyan, D. V., Koldoba, A. V., et al., MNRAS, 387, 63B

Carciofi, A. C., Bjorkman, J. E., Otero, S. A., et al. 2011, IAU Symp. Vol. 272, ed. C. Neiner, 325

Carciofi, A. C., Bjorkman, J. E. Otero, S., et al. 2012, ApJL, 744, L15

Christodoulou D. M., Laycock S. G. T., Yang J., Fingerman S., 2016, ApJ, 829, 30

Corbet R. H. D., 1996, ApJ, 457L, 31C

Corbet R. H. D., 1984, A\&A, 141, 91

Dubus, G. 2013, A\&ARv, 21, 64D (D13)

Eckström, S., Georgy, C, Eggenberger, P., et al. 2012, A\&A, 537 A146E

Hamaguchi, K., Oskinova, L., Russell, C., et al. 2016, ApJ, 832, $140 \mathrm{H}$

Haubois, X., Carciofi, A. C., Rivinius, T., et al. 2012, ApJ, 756, 156

Henry, G. W. \& Smith, M. A. 2012, ApJ, 760, 10H (HS12)

Knigge, C., Coe, M. J., \& Podsladlowski, P., 2012, Nature, 479, $372 \mathrm{~K}$ 
Liu , Q. Z., van Paradijs, J., \& Van den Heuvel, E. P. J. 2006, A\&A, 455, 1165L

Lopes de Oliveira, R, Smith, M. A., \& Motch, C. 2010, A\&A, 512, A22L (LSM10)

Lutovinov, A., Tsygankov, S., \& Chernyakova, M. 2012, MNRAS, 423, 1978L (LTC12)

Manchester, R. N. 2005, Ap\&SS, 297, 101M (M05)

Motch, C., Lopes de Oliveira, R., \& Smith, M. A. 2015, ApJ, 806, 177M (MLS15)

Okazaki, A., \& Negueruela, I. 2001, A\&A, 377, 161 O

Okazaki, A. 2011, priv. commun.

Özel, F., Psaltis, D., Ramesh, N., et al. 2012, A\&A, 757, 55 O

Özel, F., \& Freire, P. 2016, ARA\&A, 54, 401O

Özel, F., \& Freire, P. 2016, ARA\&A, 54,401O ans

Popov, S.B., \& Turolla, R. 2012, MNRAS, 421, L127 (PT12)

Portegies Zwart, S., \& Verbunt, F. 1996, A\&A, 309, 179P (PV96)

Porter, J. M. 1996, MNRAS, 280L, 31P

Postnov, K., Oskinova, L., \& Torrejón, J. M. 2017, MNRAS, 465L, 119P (POT17)

Reig, P. 2011, Ap\&SS, 332, 1R

Reig P., Milonaki F., 2016, A\&A, 594, A45

Robinson, R. D., \& Smith, M. A. 2000, ApJ, 540, 474R (RS00)

Robinson, R. D., Smith, M. A., \& Henry, G. W. 2002, ApJ, 575, 435R (RSH02)

Shao, Y., \& Li, X.-D. 2014, ApJ, 796,37S (SL14)

Smith, M. A., Cohen, D. H., Gu, M. G., et al. 2004, ApJ,600, 972S (S04)

Smith, M. A., Lopes de Oliveira, R., \& Motch, C., et al. 2012, A\&A, 540, A53S (SLM12a)

Smith, M. A., Lopes de Oliveira, R., \& Motch, C. 2012b, ApJ, $755,64 \mathrm{~S}$ (SLM12b)

Smith, M. A., Lopes de Oliveira, R., \& Motch, C., 2016, AdSpR, $58,782 \mathrm{~S}$ (SLM16)

Smith, M. A., \& Robinson, R. D. 2003, in "Interplay of Periodic, Cyclic, and Stochastic Variability," ASP Conf. Ser. 292, 263S (SR03)

Smith, M. A., Robinson, R. D., Corbet, R. H. 1998a, ApJ, 503, 877S (SRC98)

Smith, M. A., Robinson, R. D., Hatzes, A. P.. 1998b, ApJ, 508, $945 \mathrm{~S}$

Stee, Ph., Delaa, O., Monnier, J., et al. 2012, A\&A, 545A, 59S

Torrejón, J. M., Schulz, N. S., \& Nowak, M. A. 2012, ApJ, 750, $75 \mathrm{~T}$

Tsygankov, S., Lutovinov, A., Doroshenko, V., et al. 2016, A\&A, $593 \mathrm{~A}, 16 \mathrm{~T}$

Waters L. B. F. M., van Kerkwijk M. H., 1989, A\&A, 223, 196

This paper has been typeset from a $\mathrm{T}_{\mathrm{E}} \mathrm{X} / \mathrm{LAT} \mathrm{E}$ file prepared by the author. 